\title{
Diversity of Primate Lentiviruses Rebooted
}

\author{
Jayashree S. Nandi'1, Anil K. Chhangani², Shravan Singh Rathore3, Bajrang Raj J. Mathur4 \\ ${ }^{1}$ Department of Microbiology \& Immunology, Albert Einstein College of Medicine, New York, USA \\ ${ }^{2}$ Department of Environmental Science, Maharaja Ganga Singh University, Bikaner, India \\ ${ }^{3}$ Department of Wildlife, Machiya Biological Park, Jodhpur, India \\ ${ }^{4}$ Department of Veterinary Sciences, Patanjali Products 1-B-1, Kamla Nehru Nagar, Behind Konark School, Jodhpur, India \\ Email: jseemanandi@gmail.com,chhanganiak@mgsubikaner.ac.in,rathore_srvn@yahoo.com,bajrangrajmathur@gmail.com
}

How to cite this paper: Nandi, J.S., Chhangani, A.K., Rathore, S.S. and Mathur, B.R.J. (2019) Diversity of Primate Lentiviruses Rebooted. Journal of Biosciences and Medicines, 7, 126-138.

https://doi.org/10.4236/jbm.2019.712011

Received: October 10, 2019

Accepted: December 7, 2019

Published: December 10, 2019

Copyright $\odot 2019$ by author(s) and Scientific Research Publishing Inc. This work is licensed under the Creative Commons Attribution International License (CC BY 4.0).

http://creativecommons.org/licenses/by/4.0/

\begin{abstract}
Highlight: The present report reveals for the first time natural lentiviral infection of wild Indian NHPs, rhesus monkeys (Macaca mulatta) and langurs (Semnopithecus entellus) by SIVs that are phylogenetically diverse from all known SIVs, including "SIVmac", which infects captive rhesus monkeys. The novel SIVs are intriguingly homologous to HIV-1, based on serology and partial lentiviral genomic sequence analyses. Diverse lentiviruses infect human and nonhuman primates (NHPs). There are more than 45 different "species-specific" simian immunodeficiency viruses (SIVs) that infect their cognate NHP hosts in natural habitats in Africa. Indian NHPs are not known to be infected by SIVs in the wild. Conventionally SIVs are named after their natural hosts, except for SIVmac, which infects captive rather than wild rhesus macaques. SIVmac is therefore a misnomer. It is a genetic variant of the African SIVsmm, which infects wild African sooty mangabey monkeys. SIVsmm is the progenitor of human immunodeficiency virus (HIV-2), while SIVcpz that infects wild chimpanzees is the progenitor of HIV-1. Although natural infections cannot be easily studied in wild NHP populations, we have previously reported co-infection of wild Indian NHPs by other retroviruses: simian retroviruses (SRVs) and Simian Foamy viruses (SFV). Apart from zoonosis, transmission of pathogens from humans to animals: anthroponosis, has also been reported in literature.
\end{abstract}

\section{Keywords}

Lentivirus, HIV-1, SIV, Wild Indian Langur, Rhesus Monkey, Zoonosis, Anthroponosis

\section{Introduction}

Diverse lentiviruses infect human and nonhuman primates (NHPs). Zoonotic 
pathogens represent $60 \%$ of all known human pathogens [1]. Crossspecies transmission has led to many devastating epidemics which remains a poorly understood phenomenon. Presence of zoonotic pathogens in humans relies on human-animal interface and their environments. The interhuman barrier determines the ability of zoonotic pathogens to efficiently transmit among humans, causing outbreaks, epidemics, or pandemics [2]. Once cross-species transmission of pathogens has occurred, interhuman transmission maintains the infection in the population. For successful cross species transmission, only "spillover" into a single individual of a new host species is not sufficient. For a zoonotic virus to become endemic, sustained chain of transmission in the new host population ("host switching") is required [3].

While the history of HIV-1 as a result of zoonosis of SIVcpz, and HIV-2 due to zoonotic transmission of SIVsmm is documented, there are over 45 different simian lentiviruses that infect different species of African NHPs, but human equivalent of these SIVs are not reported. Bushmeat hunting and consumption constitutes the primary risk factor for zoonotic disease transmission of simian retroviruses including SIVs in Africa.

According to the current understanding, lentiviruses do not infect Indian NHPs in the wild [4]. The present report however reveals natural lentiviral infection of wild Indian NHPs, rhesus monkeys (Macaca mulatta) and langurs (Semnopithecus entellus) based on serology and partial lentiviral genomic sequences. The SIVs are phylogenetically diverse from all known African SIVs, including SIVmac that infects captive rhesus monkeys and are intriguingly homologous to HIV-1. Exogenous lentiviruses appear to have far more extensive history of host switching than previously appreciated.

Earlier we reported co-infection of wild Indian NHPs from forested regions of Rajasthan State by other exogenous retroviruses: Simian Foamy viruses (SFV) and Simian retroviruses, SRV-6 and SRV-7 [5] [6] [7].

Human-wildlife interaction and conflict in India are different from the African scenario and bushmeat consumption is not common here. The notion that Indian NHPs are not infected by SIVs is based on negative results of sero-surveillance for lentiviral infection tested in plasma samples from numerous wild Indian NHPs during the early phase (1980's) of AIDS pandemic by older, less sensitive serological assays.

Chimpanzees, gorillas, baboons or sooty mangabey monkeys are not found in India, but a large number of different NHP species are distributed here. Export of wild rhesus monkeys from India was discontinued in 1978.

Indian rhesus monkeys currently used in the West are captive bred that are used as experimentally infected primate models to investigate various aspects of AIDS/HIV infection and anti-viral therapy, vaccine etc. Indeed, humans and rhesus monkeys are known to be "non-natural hosts" for lentiviruses, HIV and SIV.

Because of the religious connotation of simians among the Hindus in India, 
there has been a close man-monkey interaction for centuries. Rapid urbanization and deforestation have resulted in fragmentation of natural habitats of the feral simians, so they frequently move near human dwellings in search of food.

Rhesus monkeys belonging to subfamily cercopithecae and langurs, to colobinae subfamily of Old World monkeys (OWM) are widely distributed in India [8]. Occasionally, the simians aggressively attack, bite and scratch unsuspecting humans to grab food items. Such interactions can lead to zoonosis as well as anthroponosis, when infected blood or saliva from the human and NHP hosts are inadvertently mixed. This can lead to the emergence of unreported infectious diseases [9]. As the human-animal connection escalates, so does the threat for pathogen spread.

Host restriction factors: There are several host proteins that restrict replication of viral pathogens. The tripartite motif (TRIM) family of E3 ubiquitin ligases is a major contributor to host innate immunity and is involved in host-virus interaction. TRIM family members are characterized by their conserved RBCC domain that comprises the RING (R), one or two B-boxes (B), and the coiled-coil (CC) domains. The most prevalent C-terminal domain is the PRY-SPRY domain (or B30.2). The SPRY domain of TRIM5a is able to bind the capsid of HIV thereby restricting its replication. Inhibition of retroviral reverse transcription has been shown to require all domains of TRIM5 $\alpha$, each providing a unique tool for host immunity [10].TRIM5a of wild rhesus monkeys was found to be identical to equivalent sequence of captive rhesus monkeys (Figure 1(a) and Figure 1(b)). Other restriction factors against viral infection like APOBEC3, SAMDH1 and Tetherin (BST-2) are subjects for future investigation [11] [12].

\section{Materials and Methods}

Even though viral infections cannot be easily studied in wild NHP population, field studies of SIV-infected hosts are necessary to unravel the phenomenon of natural infection.

Blood samples from 35 feral rhesus monkeys from Jaipur and 9 langurs from Jodhpur were collected in 2010 by temporary trapping of the feral simians in collapsible cages, with the aim to investigate natural lentiviral infection. We obtained permission from the Department of Forest and Wildlife of Rajasthan Govt. for sample collection for the investigation. On a later field visit in 2016, blood samples from only 1 rhesus monkey and 3 langurs could be collected (Figure 1(c)). It is not possible to collect a large number of samples from the wild NHPs because the monkeys are very alert and difficult to trap and also because the local government authorities gave permission to collect only a limited number of blood samples for research purpose (30 - 40 samples per visit). The small sample size was not amenable to statistical analysis on this occasion.

Bananas and chickpeas were used as baits and the animals were trapped by 


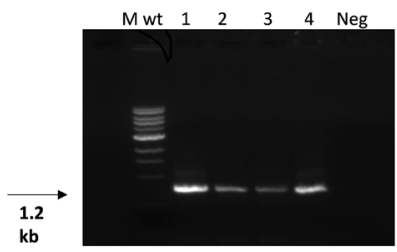

(a)

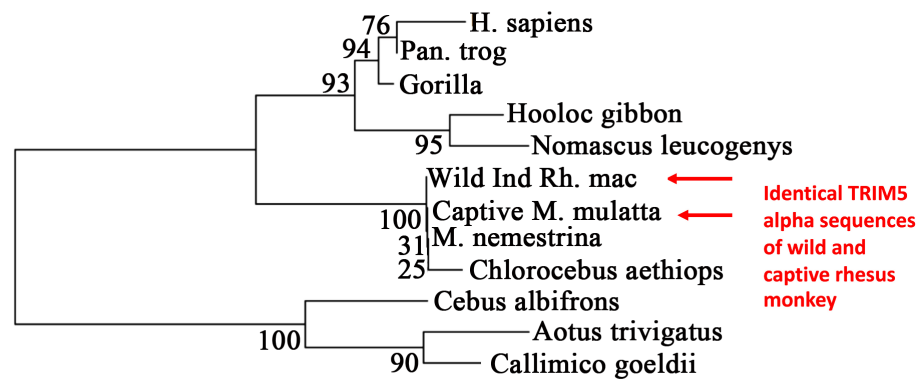

$\lcm{102}$

(b)

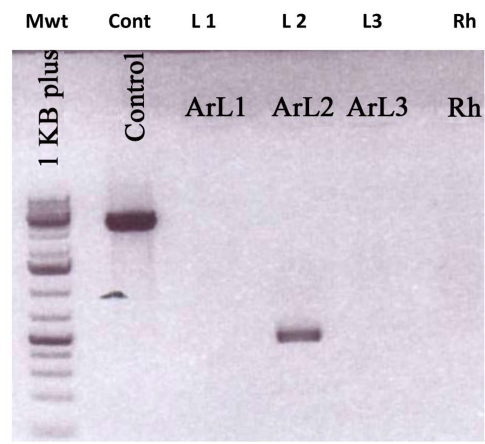

(c)

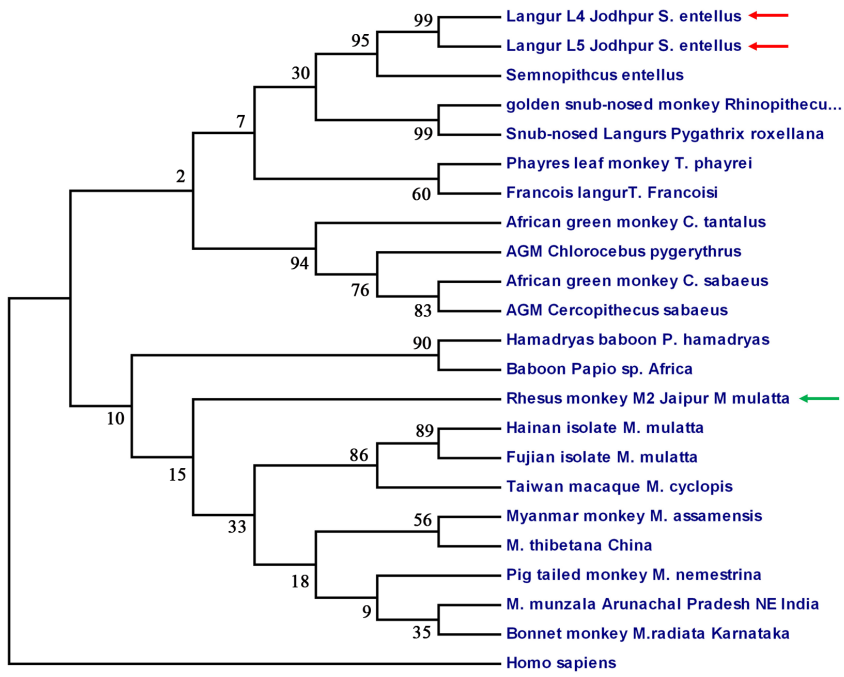

(d)

Figure 1. (a) TRIM5 alpha PCR products from wild Indian rhesus monkeys; (b) TRIM5 alpha phylogenetic tree; (c) PCR products from wild Indian primates amplified with HIV-1 primers from vif and LTR region; (d) Novel SIV infected wild Indian host primate species identified by cytochrome b mitochondrial DNA analyses. 
professional trappers. The animals were tranquillized using ketamine hydrochloride and Xylazil-100 (Troy, Australia) in 1:1 ratio in prescribed dose according to the body weight of the simians before drawing blood by local veterinary professionals. The simians were released in the natural habitat after phlebotomy.

Peripheral blood mononuclear cells (PBMC) from blood samples were purified by standard method of centrifugation on Ficoll Hypaque (Sigma, USA) and used for virus isolation by co-culture with primary human PBMC and human T cell lines, Hut 9, U87, Molt 4.

Serology: Serological screening of plasma samples with commercial HIV-1/2 Rapid test and WB assay was performed to detect cross reacting antibody to HIVs as a test for lentiviral infection. Commercial SIV serology tests were not available locally so HIV-1/2 serology tests were used to analyze the simian plasma samples. Such diagnostic approach has been used for screening of plasma samples of African NHPs [13]. Immunoblot test was also performed with specific monoclonal antibody against HIV-1 p24 antigen and SIVmac p27 antigen for detection of HIV/SIV specific antigens.

Extraction of DNA and RNA: DNA was extracted from peripheral blood mononuclear cells (PBMC) using Tri reagent (MRC Inc. Cincinnati). Plasma samples were used for extraction of viral RNA using QIA amp viral RNA kit (Qiagen, California) for subsequent DNA PCR and RT-PCR assays. PBMC was also used for virus isolation by co-culture with primary human PBMC from healthy individuals as well as human T-cell lines. Purified PBMC and plasma samples were labeled and stored in screw capped tubes in deep freezes $\left(-80^{\circ}\right)$ until shipment to the USA on dry ice by designated shipping Company with necessary permit from the Center for Disease Control and Prevention (CDC), Atlanta (USA) and US Department of Fisheries and Wildlife (USFWS). Export permit was obtained by the Veterinary authorities of Rajasthan.

Laboratory experiments: After the collection of samples at the field sites, downstream laboratory experiments and sequencing of partial lentiviral genes were performed at the HIV Program of the National Cancer Institute, Frederick, MD. TRIM5 $\alpha$ related investigation was performed at the Dept of Microbiology \& Immunology of the Albert Einstein College of Medicine, Bronx, NY.

Amplification of viral genomic by Polymerase Chain Reaction (PCR): To molecularly characterize the lentiviruses infecting wild Indian langurs and rhesus monkeys, partial lentiviral genes were amplified by PCR, DNA PCR to detect integrated proviruses and RT-PCR for detection of replicating lentiviruses.

All primate lentiviruses share three structural genes: group specific antigen, gag, viral polymerase, $p o l$ and envelope gene, env, which encode the basic structural and enzymatic proteins required for infection and replication. Sequences of gag and polgenes are relatively conserved but the env genes are variable and are primarily involved in interaction with the host target immune cells. Additionally, the lentiviruses possess a variable repertoire of accessory genes: nef, vif and $v p r$ present in all primate lentiviruses, while vpu is only found in certain viruses, such as SIVcpz, SIVgor (infecting gorillas), and HIV-1. Similarly, the accessory 
gene $v p x$ is only found in lentiviruses, such as SIVsm, SIVmac and HIV-2. Regulatory genes tat and rev are also involved in replication of both HIV and SIV. While the accessory proteins are not required for basic replication of the virus in most cell lines, their importance in vivo has been proven by both experimental and naturally occurring accessory gene deletion mutants [14].

In the first instance, degenerate primers from the conserved RT region of the lentiviral pol gene were employed that could amplify both SIV and HIV genomes. Other lentiviral genomic regions that were also amplified using relevant primer pairs designed from sequence information from the Los Alamos National Laboratory (LANL) HIV database (US), included the structural genes gag and env as well as the lentiviral accessory genes vif, vpr, nef and LTR region. The amplicons were directly sequenced by Sanger sequencing, performed by commercial Company (Macrogen, USA). Sequences of the lentiviruses have been deposited in the Genbank data base. The accession numbers are provided at the end of the article.

Viral load: Viral load in plasma samples of representative langurs and rhesus monkeys was determined independently in coded samples by sensitive qRT-PCR using primers and probe from gag region of Subtype B HIV-1. The values ranged from undetectable, low or medium range (Table 1 ).

Partial lentiviral gene sequences were next used for phylogenetic analyses using standard genetic software programs. Prior to the phylogenetic analyses, nucleotide substitution saturation was tested in Dambe by plotting the observed transitions and transversions versus the evolutionary distance. Due to saturation

Table 1. Viral load assay of feral rhesus macaques (M) and langurs (L) performed independently.

\begin{tabular}{ccc}
\hline Simian species & Sample Number & Viral Load copies/mL \\
\hline Langur (Semnopithecus entellus) & L1 & \\
L2 & L3 & 6930.0 \\
Lhesus macaque (Macaca mulatta) & L4 & $*<33$ \\
L5 & L6 & 7956.7 \\
M1 & 880 \\
M2 & 2346.7 \\
M3 & 953.3 \\
M4 & $* *<33$ \\
M5 & 990 \\
& & $* *<33$ \\
& & 1870.0
\end{tabular}

${ }^{*}$ L1: Insufficient sample; ${ }^{*}$ : Below limit of detection. Viral load assay was performed independently in coded simian plasma samples by the Core Virology laboratory of the HIV Drug and Replication program of the National Cancer Institute, Frederick, MD by a sensitive quantitative RT-PCR assay. VL was undetectable in langur sample L2 and rhesus macaque samples M2 and M4. Langur sample L4 and rhesus macaque sample M5 were subjected to molecular assay for viral genomes since they had relatively higher VL values. 
at the nucleotide sequence level, phylogenetic analyses of the generated sequence data were conducted with the predicted amino-acid sequences using various algorithms.

Sequences from the rhesus macaque and langurs were compared with reference sequences (downloaded from the LANL database) of HIV-1 and SIV LTR, Gag, Nef and Env.

As the monkey species included in the study were from north India, we downloaded HIV-1 B and HIV-1C sequences from Northern India and China. Published reports indicate common source of HIV-1subtype B infection India [15] and viruses of subtype B HIV-1 from China are the main source of $B / C$ recombinant in India [16]. Multiple sequence aliments were performed in AliView v1.17.1 [17]. Maximum likelihood phylogenetic analysis was performed in FastTreev1 and confirmed in MEGA6 [18].

\section{Result}

The SPRY domain of TRIM5 $\alpha$ of wild and captive rhesus monkeys were found to be identical and different from the host factor from other species of NHPs (Figure 1(a) and Figure 1(b)).

Screening of simian plasma samples by serology revealed the presence of antibody that cross reacted with HIV-1 antigen. A total of 15 rhesus macaques out of 35 samples analyzed (45\%), and 6 of 9 (66.6\%) langur samples were found to be seropositive by HIV-1WB assay. In the collection of 2016, the lone sample from rhesus monkey was negative by serological and PCR assay (Figure 1(c)). Two of 3 langur samples were sero-reactive, while 1 langur sample gave a distinct product amplified with primers from vif and 3'LTR region that was smaller than the control HIV-1 product, probably representing a quasispecies (Figure 1(c)). Mitochondrial DNA analyses confirmed the simian host species of origin, rhesus monkey and langur (Figure 1(d)). Extreme precaution was taken at every step of laboratory experimental process to avoid laboratory contamination during PCR experiments. The reference strain HXB2of subtype B HIV-1was not used in any of the experiments.

When tested by specific immunoblot assay, 15/35 rhesus monkeys and 6 of 9 langur samples revealed the presence of antigens that cross reacted with HIV-1 Gag monoclonal antibody, p24, but similar assay with SIVmac p27 monoclonal antibody were seronegative (data not shown).

Molecular characterization of the novel SIVs: We first performed phylogenetic analysis of the conserved gag genes (Figure 2(a)). In the predicted amino acid Gag sequences, the novel SIVs revealed unexpected homology to HIV-1subtype B sequences from north India with $>80 \%$ bootstrap support (subtype B HIV-1, Genbank Accession no: EF694037).

Partial Gag protein of the SIVs infecting wild rhesus macaques clustered together with $>74 \%$ bootstrap support. One sequences from Langur clustered with subtype B HIV-1sequences from China (Figure 2(a)). In the phylogenetic tree 


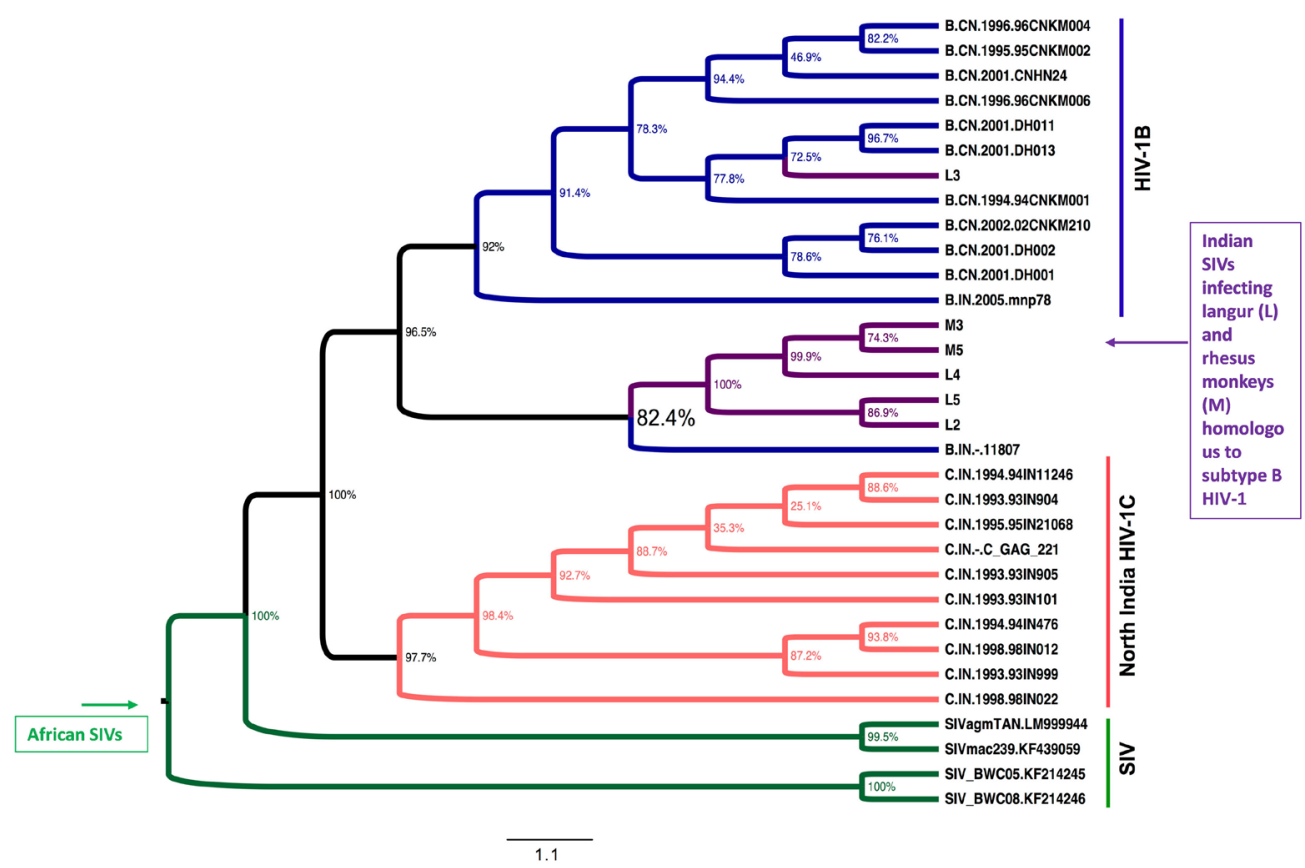

(a)

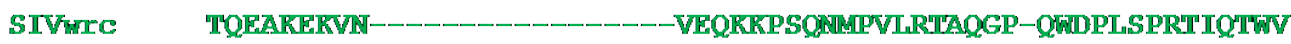
SIV langur TRRAADRIIEEQNRSKRKAQQAAADTGHSNQVSQNYPIVQNIQGQMVHQAISPRITINAWV $\star: \star \star: \star:: \quad \ldots:: \star \star \star \star:::$ :

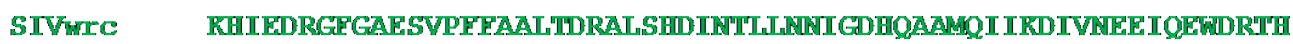
SIV langur RVVEERAFSPEVIPMF SALSEGATPODLWTIMINTVGGHQAAMOMIRETINEEAAEWDRVH

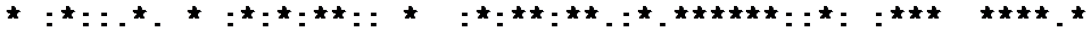

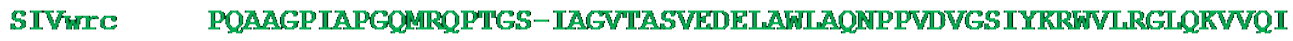

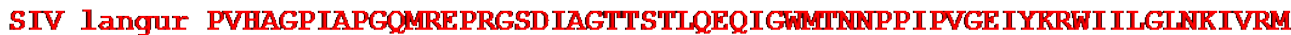

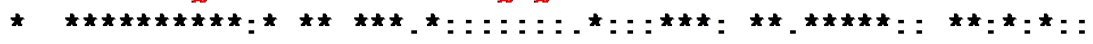

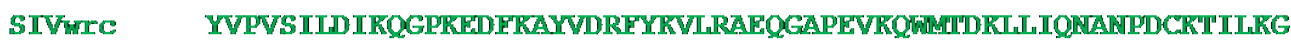
SIV langur YSPTSIIDIRQGPREPFRDYVDRFYRTIRAEQASQEVRNWMITETLVONANPDCKTIIKA

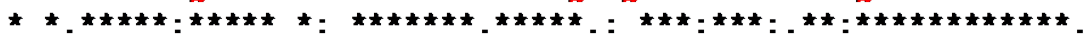

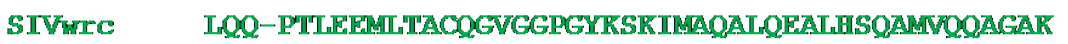
SIV langur LGPAATIEEMMTIPCQGVGGPGHRARVLAEAMSQVITSATIMMQRGN-

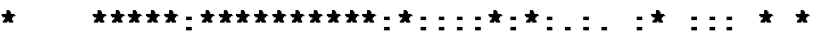

(b)

$\begin{array}{rrrrr}10 & 20 & 30 & 40 & 50\end{array}$

\begin{tabular}{|c|c|}
\hline HXB2 & SVITQACPKVSFEPIPIHYCAPAGFAILKCNNKTFNGTGP-C \\
\hline L4 & .F.................... \\
\hline M2 & 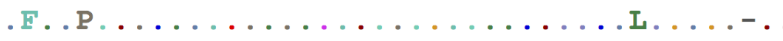 \\
\hline M5 & 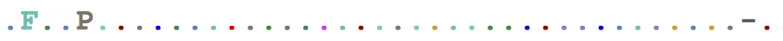 \\
\hline \multirow[t]{3}{*}{ SIVmac. DQ136310 } & . . QES.D.HYWDA.RFR . . P.Y.L.R. DTNYS . FM.K \\
\hline & 80 \\
\hline & $\begin{array}{l}\ldots|\ldots| \ldots|\ldots| \ldots|\ldots| \ldots|\ldots| \ldots|\ldots| \ldots \\
\text { THGIRPVVSTOLLINGSLAEEEVVIRSVNFTDNAKTIIVQLN }\end{array}$ \\
\hline L4 & $\ldots \ldots$ AL.........D.G............ \\
\hline M2 & $\ldots \ldots \ldots \ldots \ldots \ldots$ D. . . . . . . LI. \\
\hline M5 & $\ldots \ldots \ldots \ldots \ldots \ldots$. . . . . . . . .LL. \\
\hline SIVma & . RMMETQT . .WFGF . . TR . NRTY .---YWHGRDNRT . IS . \\
\hline
\end{tabular}

(c) 


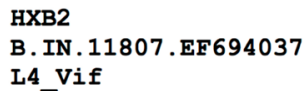

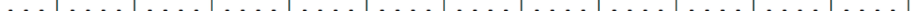
MENRWQVMIVWQVDRMRIRTWKSLVKHHMYVSGKARGWFYRHHYESPHPRISSEVHIPLG $\ldots \ldots \ldots \ldots \ldots \ldots \ldots \ldots \ldots$ I. . KK. T. K. . . . . . . . . E $\ldots \ldots \ldots \ldots \ldots$. . . R . . P . I.R. KD . K . . . TN.K. .K.P. . $\ldots \ldots \ldots \ldots \ldots$. . R . . . I.R. KD . . . . TN.K. . . . . . $\begin{array}{cccccc}70 & 80 & 90 & 100 & 110 & 120\end{array}$ $\ldots|\ldots| \ldots|\ldots| \ldots|\ldots| \ldots|\ldots| \ldots|\ldots| \ldots|\ldots| \ldots|\ldots|$ DARLVITTYWGLHTGERDWHLGQGVSIEWRKKRYSTQVDPELADQLIHLYYFDCFSDSAI E.K. . . . . . . . . . . . . E. . . . . . . . . . . . . E. . V K. . . . . . P. .KK. . . P.SPLK . . KFY.P.N.GPTK.P.P.PH .W. .K. .N

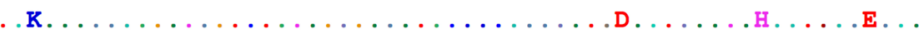
$\begin{array}{llllll}130 & 140 & 150 & 160 & 170 & 180\end{array}$ $\ldots|\ldots| \ldots|\ldots| \ldots|\ldots| \ldots|\ldots| \ldots|\ldots| \ldots|\ldots| \ldots|\ldots| \ldots|\ldots|$ RKALLGHIVSPRCEYQAGHNKVGSLQYLALAALITPKKIKPPLPSVTKLTEDRWNKPQKT

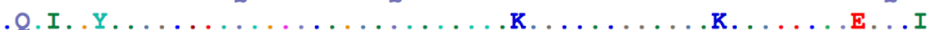
K.TI.RPNG. KG.I. T.RG . . . . . * NTI . R . . . . . . . . . . . . . . . . . . . .

(d)

Figure 2. (a) Phylogenetic tree based on Gag sequences of Indian SIVs indicate intriguing homology to HIV-1B, distinct from African SIVs (in green); (b) Alignment of Gag sequences of SIV infecting Indian langur (Colobinae), with equivalent sequences of SIV infecting African wild red colobine (SIVwrc), showing only 52\% homology; (c) Alignment of partial translated Env (gp120) sequences of novel SIVs infecting wild langur (L) and rhesus monkeys (M), homologous to HIV-1 reference sequence, HXB2 and distinct from SIVmac; (d) Alignment of translated Vif sequences of SIV infecting langur L4 with HXB2, HIV-1 reference strain and HIV-1 from India and Venezuela.

African SIV sequences including SIVmac, SIVagm, SIVsmm, clustered as separate branch, highlighted in green.

Since langurs belong to the colobinae subfamily of primates, we aligned the conserved Gag region of SIVwrc, that infects wild red colobine from Africa with equivalent sequences of SIV infecting langur L4 (Figure 2(b)). Only 52\% identity in the translated amino acid sequences of Gag protein was observed compared to nearly $84 \%$ identity with Gag p24 sequences of subtype B HIV-1 (Figure 2(a), homology with HIV-1 in purple).

African SIVs included in construction of the phylogenetic tree: Sequences of equivalent viral region of African SIVs used for homology search included SIVcpz, SIVsmm, SIVmac, SIVcol, SIVagm and SIVwrc (Figure 2(a) and Figure 2(b)). The novel Indian SIVs were different from African SIVs including SIVmac (Figure 2(c)) but significantly homologous to Indian subtype B HIV-1 isolates (Figure 2(d)).

Multiple sequence alignment of translated amino acid sequences from partial surface glycoprotein (gp120) of the env gene of SIVs infecting Indian langur L4 and rhesus monkeys M2 and M5 with subtype B HIV-1 reference strain HXB2 revealed striking homology, while equivalent sequences from SIVmac (Genbank Accession No: DQ136310) were completely different (Figure 2(c)). The results confirm that SIVmac infecting captive rhesus macaques are different from the novel SIVs infecting wild Indian rhesus macaques.

Vif sequences from SIV infecting rhesus monkeys M2, M5 and langur L4 were truncated (Figure 2(d)). The Vif region of SIVs infecting both rhesus monkeys and langurs was truncated at the 3 'end. 
Alu-LTR PCR: Integration of lentivirus in the monkey chromosome was confirmed by amplification of the integration site by Alu-LTR repeat PCR, with genomic DNA extracted from PBMC of langurs and rhesus monkey (data not shown).

\section{Discussion}

The majority of human emerging infectious diseases are zoonotic, with viruses that originate in wild mammals being of particular concern, like HIV and Ebola viruses. Understanding patterns of viral diversity and transmission in wildlife species are key goals for pandemic surveillance programs, and of vital public health importance, which is the basis for the present investigation. The study included extensive field and laboratory research work spanning over 2 decades, that started in India at the Department of Retrovirology, National Institute of Virology, Pune in 1998.

Cross-species transmission is driven by exposure and constrained by host genetic restriction factors that determine host-virus interaction in natural setting, as opposed to the experimental infection of captive NHPs in artificial conditions at primate research facilities.

The putative viral coevolution during primate speciation suggests that many of these zoonotic transmissions were ancient that have been acted on by natural selection for millions of years. The nature of the host-virus relationship is highly variable for primate lentiviruses, with some viruses switching hosts often while others maintain strict host specificity.

Infection of feral Indian langurs and rhesus monkeys by novel exogenous SIVs has not been reported before. The SIVs are not related to any African SIV but were unexpectedly homologous to subtype B HIV-1, based on experimental evidence presented here confirm that the data are clearly not speculative or biologically impossible.

The evidence for a novel animal reservoir of HIV-1 in wild Indian simian population has implications for Public Health and conservation of primate species.

In future, whole genome sequence of the novel SIV genomes would be determined by using the Next Generation Sequencing (NGS) approach for a more robust identification of these unique lentiviruses from the forests of Rajasthan.

The on-going viral dynamics, host restriction factors and virus-host interaction in wild simian population infected by multiple retroviruses are important areas of investigation.

\section{Limitations and Strengths}

The limitations of the study are the small sample size, due to reasons inherent with field conditions. The hitherto unreported findings open up a new chapter in primate lentivirus epidemiology. Additional sampling and whole genome sequencing data of the novel SIV genomes by Next Generation Sequencing (NGS) will cer- 
tainly establish the phylogenetic identity further and will be perused in future.

\section{Conclusion}

In summary, we propose a counter intuitive reverse transmission of HIV-1 from infected humans to simians through aggressive monkey bite, given the close man-monkey interaction existing in India. From the present report, it cannot be stated if the reverse transmission events represent more than one transmission from man-to-monkey or if this was a single transmission event which led to subsequent transmission between langurs and macaques living in close proximity in the natural habitat. Only further detailed epidemiological and virological studies will address these questions.

\section{Genbank Accession Numbers}

GenBank Accession numbers of generated sequences: GQ472903, GQ472907, GQ472906, GQ472914, GQ472913, GQ472918, GQ472908, GQ472910, GQ472923, GQ472921, GQ472909.1, GQ472905, GQ472906.

\section{Acknowledgements}

Financial support was provided by the Primate Research Center, Jodhpur, India, and Maharaja Ganga Singh University, Bikaner for field work, which is gratefully acknowledged. Intramural funding from National Institutes of Health, US for sequencing and phylogenetic analyses is acknowledged with thanks. Intramural funds from the Dept of Microbiology \& Immunology, Albert Einstein College of Medicine, Bronx, New York was provided for the TRIM5 related research. The authors acknowledge the help with thanks. Help of Ujjwal Niyogi, Karolinska Institute, Sweden for phylogenetic analyses of viral sequences is acknowledged with thanks.

\section{Author Contribution}

Jayashree S. Nandi conceived and initiated the investigation in 1998 in her laboratory of Retrovirology, National Institute of Virology, Pune, India, before moving to the US in 2002. She took part in the field work, all laboratory investigation including phylogenetic analyses carried out in the US and wrote the manuscript. She is currently located in Pune, India, her home country. Anil Kumar Chhangani, Shravan Singh Rathore and Bajrang Raj Mathur carried out field work, phlebotomy, and PBMC purification in Jaipur and Jodhpur (India).

\section{Conflicts of Interest}

The authors declare no conflict of interest. All authors have read and approved the manuscript.

\section{References}

[1] Olival, K.J., Hosseini, P.R., Zambrana-Torrelio, C., Ross, N., Bogich, T.L. and Das- 
zak, P. (2017) Host and Viral Traits Predict Zoonotic Spillover from Mammals. Nature, 546, 646-650. https://doi.org/10.1038/nature22975

[2] Taylor, L.H., Latham, S.M. and Woolhouse, M.E. (2001) Risk Factors for Human Disease Emergence. Philosophical Transactions of the Royal Society B, 356, 983-989. https://doi.org/10.1098/rstb.2001.0888

[3] Bell, S.M. and Bedford, T. (2017) Modern-Day SIV Viral Diversity Generated by Extensive Recombination and Cross-Species Transmission. PLOS Pathogens, 13, e1006466. https://doi.org/10.1371/journal.ppat.1006466

[4] Apetrei, C., Kaur, A., Lerche, N.W., et al. (2005) Molecular Epidemiology of Simian Immunodeficiency Virus SIVsm in U.S. Primate Centers Unravels the Origin of SIVmac and SIVstm. Journal of Virology, 14, 8991-9005. https://doi.org/10.1128/JVI.79.14.8991-9005.2005

[5] Nandi, J.S., Van Dooren, S., Chhangani, A.K. and Mohnot, S.M. (2006) A New Recombinant Beta-Retrovirus from the Feral Rhesus Monkeys (Macaca mulatta) from Jaipur Rajasthan, India. Virus Genes, 33, 107-116.

https://doi.org/10.1007/s11262-005-0032-x

[6] Nandi, J.S., Tikute, S.A., Chhangani, A.K., Potdar, V.A., Tiwari-Mishra, M., et al. (2003) Natural Infection by Simian Retrovirus-6 (SRV-6) in Hanuman Langurs (Semnopithecus entellus) from Two Different Geographical Regions of India. Virology, 311, 192-201. https://doi.org/10.1016/S0042-6822(03)00187-9

[7] Nandi, J.S., Bhavalkar-Potdar, V., Tikute, S. and Raut, C.G. (2000) A Novel Type D Simian Retrovirus (SRV) Naturally Infecting the Indian Hanuman Langur (Semnopithecus entellus). Virology, 277, 6-13. https://doi.org/10.1006/viro.2000.0567

[8] Chetan, N., Praveen, K.K. and Vasudeva, G.K. (2014) Delineating Ecological Boundaries of Hanuman Langur Species Complex in Peninsular India Using MaxEnt Modeling Approach. PLoS ONE, 9, e87804. https://doi.org/10.1371/journal.pone.0087804

[9] Messenger, A.M., Barnes, A.N. and Gray, G.C. (2014) Reverse Zoonotic Disease Transmission (Zooanthroponosis): A Systematic Review of Seldom-Documented Human Biological Threats to Animals. PLOS ONE, 9, e89055. https://doi.org/10.1371/journal.pone.0089055

[10] Morger, D., Zosel, F., Bohlmann, M., Zoger, S., Mittelviefhaus, M., Schuler, B., Luban, J. and Grotter, M.G. (2018) The Three-Fold Axis of the HIV-1 Capsid Lattice Is the Species-Specific Binding Interface for TRIM $5 \alpha$. Journal of Virology, 92, e01541-17. https://doi.org/10.1128/JVI.01541-17

[11] Krisko, J.F., Martinez-Torres, F., Foster, J.L. and Garcia, J.V. (2013) HIV Restriction by APOBEC3 in Humanized Mice. PLOS Pathogens, 9, e1003242. https://doi.org/10.1371/journal.ppat.1003242

[12] Mayte, C., Bermejo, M., Descours, B., et al. (2016) IL-7 Induces SAMHD1 Phosphorylation in CD4+ T Lymphocytes, Improving Early Steps of HIV-1 Life Cycle. Cell Reports, 14, 2100-2107. https://doi.org/10.1016/j.celrep.2016.02.022

[13] Lauck, M., Switzer, W.M., Sibley, S.D., et al. (2013) Discovery and Full Genome Characterization of Two Highly Divergent Simian Immunodeficiency Viruses Infecting Black-and-White Colobus Monkeys (Colobus guereza) in Kibale National Park, Uganda. Retrovirology, 10, 107. https://doi.org/10.1186/1742-4690-10-107

[14] Faust, T.B., Binning, J.M., Gross, J.D. and Frankel, A.D. (2017) Making Sense of Multifunctional Proteins: Human Immunodeficiency Virus Type 1 Accessory and Regulatory Proteins and Connections to Transcription. Annual Review of Virology, 4, 241-260. https://doi.org/10.1146/annurev-virology-101416-041654 
[15] Halani, N., Wang, B., Ge, Y.C., Gharpure, H., Hira, S. and Saksena, N.K. (2001) Changing Epidemiology of HIV Type 1 Infections in India: Evidence of Subtype B Introduction in Bombay from a Common Source. AIDS Research and Human Retroviruses, 17, 637-642. https://doi.org/10.1089/088922201300119743

[16] Neogi, U., Bontell, I., Shet, A., De Costa, A., Gupta, S., Diwan, V., et al. (2012) Molecular Epidemiology of HIV-1 Subtypes in India: Origin and Evolutionary History of the Predominant Subtype C. PLoS ONE, 7, e39819. https://doi.org/10.1371/journal.pone.0039819

[17] Larsson, A. (2014) AliView: A Fast and Lightweight Alignment Viewer and Editor for Large Datasets. Bioinformatics, 30, 3276-3278. https://doi.org/10.1093/bioinformatics/btu531

[18] Tamura, K., Stecher, G., Peterson, D., Filipski, A. and Kumar, S. (2013) MEGA6: Molecular Evolutionary Genetics Analysis Version 6.0. Molecular Biology and Evolution, 30, 2725-2729. https://doi.org/10.1093/molbev/mst197 